\title{
Noninvasive Cardiorespiratory Signals Analysis for Asthma Evolution Monitoring in Preschool Children
}

\author{
Javier Milagro, Javier Gracia, Ville-Pekka Seppä, Jussi Karjalainen, \\ Marita Paassilta, Michele Orini, Raquel Bailón, Eduardo Gil, Jari Viik
}

\begin{abstract}
Objective: Despite its increasing prevalence, diagnosis of asthma in children remains problematic due to their difficulties in producing repeatable spirometric maneuvers. Moreover, low adherence to inhaled corticosteroids (ICS) treatment could result in permanent airway remodeling. The growing interest in a noninvasive and objective way for monitoring asthma, together with the apparent role of autonomic nervous system (ANS) in its pathogenesis, have attracted interest towards heart rate variability (HRV) and cardiorespiratory coupling (CRC) analyses. Methods: HRV and CRC were analyzed in 70 children who were prescribed ICS treatment due to recurrent obstructive bronchitis. They underwent three different electrocardiogram and respiratory signals recordings, during and after treatment period. After treatment completion, they were followed up during 6 months and classified attending to their current asthma status. Results: Vagal activity, as measured from HRV, and CRC, were reduced after treatment in those children at lower risk of asthma, whereas it kept unchanged in those with a worse prognosis. Conclusion: Results suggest that HRV analysis could be useful for the continuous monitoring of ANS anomalies present in asthma, thus contributing to evaluate the evolution of the disease, which is especially challenging in young children. Significance: Noninvasive ANS assessment using HRV analysis could be useful in the continuous monitoring of asthma in children.
\end{abstract}

Index Terms-asthma, heart rate variability, children, inhaled corticosteroids, obstructive bronchitis.

\section{ACRONYMS}

$\begin{array}{ll}\text { ACh } & \text { Acetylcholine } \\ \text { ANS } & \text { Autonomic Nervous System } \\ \text { CA } & \text { Current Asthma } \\ \text { CA-N } & \text { Negative Current Asthma } \\ \text { CA-P } & \text { Possible Current Asthma }\end{array}$

This work was supported by grant BES-2015-073694 and project RTI2018097723-B-I00 from Ministerio de Economía y Competitividad. Also by Gobierno de Aragón (Reference Group BSICoS T39-17R) cofunded by FEDER 2014-2020 "Building Europe from Aragon", by CIBER in Bioengineering, Biomaterials \& Nanomedicine (CIBER-BBN) through Instituto de Salud Carlos III and by Tampere Tuberculosis Foundation. The computation was performed by the ICTS NANBIOSIS, specifically by the High Performance Computing Unit of CIBER-BBN at University of Zaragoza. Asterisk indicates corresponding author

J. Milagro*, E. Gil and R. Bailón are with the Biomedical Signal Interpretation and Computational Simulation (BSICoS) group at the Aragón Institute of Engineering Research (I3A), IIS Aragón, University of Zaragoza, Zaragoza, Spain, and CIBER de Bioingeniería, Biomateriales y Nanomedicina (CIBERBBN), Madrid, Spain, (e-mail: milagro@unizar.es).

J. Gracia and J. Viik are with the Faculty of Medicine and Health Technology, Tampere University, Tampere, Finland.

V-P. Seppä is with Revenio Research Ltd., Vantaa, Finland.

J. Karjalainen and M. Paassilta are with the Allergy Centre of the Tampere University Hospital, Tampere, Finland.

M. Orini is with the Institute of Cardiovascular Science, University College of London, London, United Kingdom.

\author{
CA-Y Positive Current Asthma \\ CRC Cardiorespiratory Coupling \\ ECG Electrocardiogram \\ HF High Frequency \\ HR Heart Rate \\ HRV Heart Rate Variability \\ ICS Inhaled Corticosteroids \\ IP Impedance Pneumography \\ LF Low Frequency \\ NN Normal-to-Normal \\ pNN50 Percentaje of successive Normal-to-Normal \\ differences greater than $50 \mathrm{~ms}$ \\ PNS Parasympathetic Nervous System \\ RMSSD Root Mean Square of Successive Differences \\ RSA Respiratory Sinus Arrhythmia \\ RTC Rapid Thoracoabdominal Compression \\ SDNN Standard Deviation of Normal-to-Normal intervals \\ SDSD Standard Deviation of Successive Differences \\ SNR Signal-to-Noise Ratio \\ SPT Skin Prick Test \\ TF Time-Frequency \\ TFC Time-Frequency Coherence \\ TVIPFM Time-Varying Integral Pulse Frequency \\ Modulation
}

\section{INTRODUCTION}

$\mathbf{L}$ UNG function assessment remains essential for the diagnosis and monitoring of several respiratory affections such as chronic obstructive pulmonary disease or asthma. Whereas in the former pulmonary function is permanently reduced, asthma is characterized by a variable and irregular respiratory tract obstruction, and therefore a continuous monitoring of airway function would be desirable in asthmatics. Yet, assessment of asthma is performed through the evaluation of the clinical history and single-time airway function measurements, being spirometry the most extended test. However, although children as young as 5 years old can perform acceptable tests, most infants and younger children are not able to conduct repeatable expiratory maneuvers, since they remain very effort dependent and require cooperation [1]. Although there are studies suggesting that children can generate acceptable flow-volume curves from an age of 3 years [2], [3], measurement criteria are to be standardized, as differences in the relative size of airways and lungs with respect to adults make the most common measurements unsuitable for this population [3], [4]. Moreover, the training 
of those children for performing the maneuvers is timeconsuming. Other lung function testing methods such as rapid thoracoabdominal compression (RTC) and raised volume RTC have been found to discriminate between health and disease [5], [6], although reference values are not yet available to be used in clinical settings [7]. For these reasons, diagnosis of asthma in young children is very dependent on the clinical history, which is retrospective in nature and could be even incomplete.

Treatment of asthma during childhood is equally challenging, and although inhaled corticosteroids (ICS) remain the standard medication for the prevention of symptoms, there is some controversy regarding the possible negative effects that ICS may have during childhood, namely growth reduction during the first weeks of treatment, or hypothalamic-pituitaryadrenal axis suppression [8]-[10]. This, together with the low perceived risk, have contributed to a low adherence to asthma treatment [11]. However, early intervention is crucial, since lung function increases up to 20 -fold during the first 10 years of life [12], so that absence of treatment when needed could lead to permanent airway remodeling [13]. In this way, a proper monitoring of the symptoms is needed in order to decide whether continuing or interrupting the ICS treatment.

These difficulties in the diagnosis, monitoring and treatment of asthma during childhood have motivated several studies aiming at developing a noninvasive tool that can be used for patient state assessment in a time-continuous manner. Most of them have focused on autonomic nervous system (ANS) monitoring, since abnormal ANS activity has been related with the pathogenesis of asthma [14], [15]. Particularly, the parasympathetic branch of the ANS is involved in bronchoconstriction [16] and bronchomotor tone control [17], and the fact that sympathetic innervation is sparse in the small airways [16] has pointed to parasympathetic nervous system (PNS) as strongly related with altered airway tone in asthmatics. Apart from the respiratory system, ANS activity also modulates cardiac activity. Thus, heart rate variability (HRV) analysis is acknowledged as a convenient tool for ANS activity assessment, and has been employed for its characterization in asthma, both in adults [18], [19] and children [15], [20]. Given the close existing relationship between HRV and respiration control, cardiorespiratory coupling (CRC) has also been suggested to be altered in some respiratory disorders [21], although to the best of our knowledge it has not been studied in asthma. Hence, the present study aims at assessing the possible clinical value of HRV and CRC analysis in the monitoring of asthma/risk of asthma evolution in children under and after ICS treatment. Since results of previous studies using HRV analysis for asthma severity/risk assessment in children suggest increased [15] and more regular [20], [22] PNS activity in subjects with worse outcomes, it is expectable that PNS activity will turn normal after treatment in subjects without or with low risk of asthma, but it should remain unchanged in children with or at high risk of asthma.

\section{MATERIALS AND MethodS}

\section{A. Study population}

The dataset employed in this study is composed of 68 children ( 45 boys and 23 girls) with a median age of 2.5 years (range $[0.9,5.7])$, who visited the Tampere University Hospital emergency room due to recurrent obstructive bronchitis. All of them were prescribed ICS treatment for three months. Electrocardiogram (ECG) and impedance pneumography (IP) were acquired at a sampling rate of $256 \mathrm{~Hz}$ with a custom designed recording device developed at Tampere University [23]. Three different recordings were scheduled for each subject:

- Recording 1 (R1) was scheduled 1 week before the end of the ICS treatment. It started at the clinic, where the parents were instructed how to place the electrodes and start and pause the biosignals acquisition, and lasted until the next morning, when parents stopped the measurement.

- Recording 2 (R2) was conducted 1-2 weeks after the end of the treatment and was performed at home, solely by the parents. In order to ensure the validity of the recordings, the parents were requested to take pictures of the electrodes placement. The recording started along the evening, and lasted until the children woke up in the morning.

- Recording 3 (R3) was scheduled 3-4 weeks after the end of the treatment and, as in $\mathrm{R} 2$, it was conducted by the parents, who were asked to take pictures of the electrodes position and to start the recording during the evening and to finish it in the next morning.

Additionally, parents were requested to annotate the times when children fall asleep and woke up in the morning.

Patients were followed up during a period of 6 months after R3 by a pediatric pulmonologist, in order to determine their current asthma (CA) status. They were labeled as having current asthma (CA-Y) if they had been prescribed medication for the control of asthma in that period because of wheezing evidence, or reported nocturnal or exercise-induced shortness of breath or cough which were reversible with bronchodilator medication. Patients who did not meet the previous criteria but were intermittently prescribed the medication because of symptoms of asthma were labeled as showing possible current asthma (CA-P), whereas all the remaining patients were labeled as showing absence of current asthma (CA-N). Patients were also classified depending on their atopic status, measured through a skin prick test (SPT). The responses to the SPT were considered positive when at least one of the assessed allergens (egg, cat, dog, birch and timothy) caused a wheel with a diameter greater than or equal to $3 \mathrm{~mm}$ without showing reaction to a negative control substance. Patients were classified as atopics or non-atopics if showing a positive or negative response to the SPT, respectively. Furthermore, they were classified based on their response to ICS treatment as effective, partially effective or not effective. The classification of the subjects attending to the different criteria is summarized in Fig. 1. Classification criterion was absent for one subject. Additionally, atopic condition was not available for one subject. 


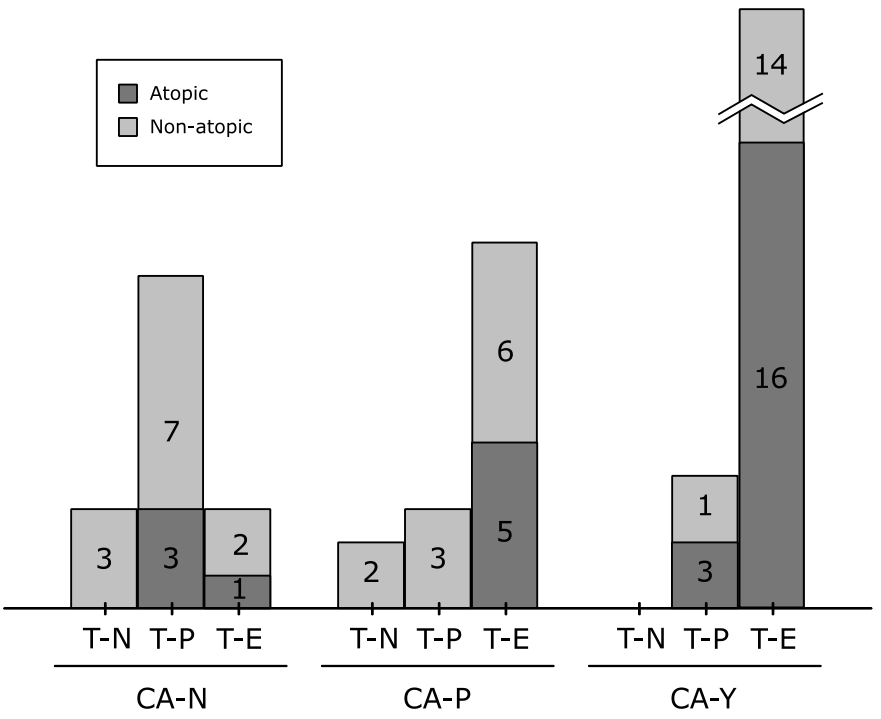

Fig. 1. Distribution of the subjects attending to the three different classifications. In the figure, CA-N, CA-P and CA-Y refer to the current asthma status (CA-N: no current asthma, CA-P: possible current asthma, CA-Y: current asthma), whereas T-N, T-P and T-E refer to the response to ICS treatment (T-N: not effective, T-P: partially effective, T-E: effective). This information was not available for 2 of the 68 subjects.

None of the subjects had previous history of laryngeal disease, tracheobronchial malacia, parechymal lung disease or bronchopulmonary dysplasia. Since these conditions might be accompanied by an asynchrony of the chest wall motion or changes in the venous return and blood volume during a breathing cycle, they could lead to a loss of linearity between the flow signal and the acquired IP signal due to changes in the electrical conductivity of the thorax, and hence to lower quality IP recordings [24]. Written informed consent was received from the parents of all the children.

\section{B. Preprocessing}

Only data recorded when the children were asleep (according to parents annotations) were analyzed, since vagal modulation of cardiac activity [25] and bronchoconstriction [26] are increased during night, together with a reduction in airway function which is specially noticeable in asthmatics [26], [27].

Preprocessing was very similar to that proposed in our previous work [20]. First, ECG signals were resampled at 1000 $\mathrm{Hz}$ with linear interpolation, so that the effect of sampling frequency on HRV analysis was reduced [28]. Afterwards, baseline wander was corrected by subtracting the ECG baseline (extracted with low-pass filtering with $0.5 \mathrm{~Hz}$ cut-off frequency) from the interpolated signals. For R-peak detection, the wavelet-based approach described by Martínez et al. [29] was employed, and the method proposed by Mateo and Laguna [30] was used for ectopic beats detection and correction. However, ectopic beats are not very frequent in young children [31], [32], so it is important to distinguish their effect from the effect of respiratory sinus arrhythmia (RSA) episodes, which are stronger for children than for adults [33]. In this way, when a beat was classified as ectopic, the following condition was checked:

$$
\theta \cdot \mathrm{RR}_{\mathrm{e}} \geq \mathrm{RR}_{\mathrm{e}-1},
$$

being $R_{\mathrm{e}}$ the time interval between the beat classified as ectopic and the previous one, $\mathrm{RR}_{\mathrm{e}-1}$ the time interval between the beat previous to the ectopic and the previous one, and $\theta$ a given threshold [20]. If the condition in Eq. 1 was satisfied, the analyzed inter-beat interval was considered to be produced by strong RSA, and the beat was not classified as an ectopic. In this work, $\theta$ was selected as 1.5 , being it adjusted manually for 6 subjects.

On the other hand, IP recordings were downsampled at 4 $\mathrm{Hz}$, and band-pass filtered (cut-off frequencies of 0.05 and 0.5 $\mathrm{Hz}$ ) so that the baseline and other components unrelated with respiration were discarded. Respiratory rate, $F_{\mathrm{r}}$, was estimated from the IP signals in a time-continuous basis, according to the method proposed by Lázaro et al. [34].

In order to avoid noisy signal segments (probably movement artifacts) that could compromise the analysis, signal to noise ratio (SNR) was evaluated beat by beat, as described by Bailón et al. [35]. Beats with a SNR more than $20 \mathrm{~dB}$ below the median SNR of the whole night recording were labeled as "low quality". If "low quality" beats were found during more than 3 consecutive seconds, this segment was considered noisy and discarded from the analysis.

\section{Heart rate variability analysis}

Time and frequency domain HRV analyses were considered, and they were performed in five-minute windows, with fourminute overlap. For the time domain analysis, mean normal-tonormal interval $(\overline{\mathrm{NN}})$, standard deviation of normal-to-normal intervals (SDNN), standard deviation of successive differences (SDSD), root mean square of successive differences (RMSSD) and percentaje of pairs of consecutive normal-to-normal intervals differing more than $50 \mathrm{~ms}$ (pNN50) were derived from the RR interval series after ectopic correction, as suggested by the Task Force [36]. For frequency domain analysis, the time-varying integral pulse frequency modulation (TVIPFM) model was employed for estimating the modulating signal, $m(t)$, which represents the autonomic modulation of the heart rate (HR) [37]. Essentially, the modulating signal is defined as:

$$
m(t)=\frac{d_{\mathrm{HR}}(t)-d_{\mathrm{HRM}}(t)}{d_{\mathrm{HRM}}(t)},
$$

where $d_{\mathrm{HR}}(t)$ stands for the instantaneous HR signal obtained from the TVIPFM model and $d_{\mathrm{HRM}}(t)$ is a low-pass filtered version ( $0.03 \mathrm{~Hz}$ cut-off frequency) of $d_{\mathrm{HR}}(t)$. The signal $m(t)$ was finally resampled at $4 \mathrm{~Hz}$.

Welch's periodogram (50-second Hamming windows, $50 \%$ overlap) was applied to $m(t)$ for estimating the HRV power spectral density corresponding to the $k$-th five-minute window, $\hat{S}_{\mathrm{HRV}}(k, F)$, and the resulting spectra were employed for frequency domain HRV analysis. Low-frequency (LF) and high-frequency (HF) power $\left(\mathrm{P}_{\mathrm{LF}}(k)\right.$ and $\mathrm{P}_{\mathrm{HF}}(k)$ respectively) were calculated from each segment. Whereas the former was 
obtained as the power within the classical $[0.04,0.15] \mathrm{Hz} \mathrm{LF}$ band [36], the frequency band used in the computation of the latter was redefined. In this way, instead of the classical [0.15, $0.4] \mathrm{Hz} \mathrm{HF}$ band, a $0.15 \mathrm{~Hz}$ bandwidth centered in the mean respiratory rate of each segment was employed [20]. The use of a modified HF band was encouraged by the increased $F_{\mathrm{r}}$ observed in children, with values close to the upper limit of the classical HF band that could lead to an underestimation of $\mathrm{P}_{\mathrm{HF}}$ due to a shift of power towards frequencies higher than $0.4 \mathrm{~Hz} . \mathrm{P}_{\mathrm{LF}}$ to $\mathrm{P}_{\mathrm{HF}}$ ratio $\left(\mathrm{R}_{\mathrm{LF} / \mathrm{HF}}(k)=\mathrm{P}_{\mathrm{LF}}(k) / \mathrm{P}_{\mathrm{HF}}(k)\right)$ and normalized LF power $\left(\mathrm{P}_{\mathrm{LFn}}(k)=\mathrm{P}_{\mathrm{LF}}(k) /\left(\mathrm{P}_{\mathrm{LF}}(k)+\mathrm{P}_{\mathrm{HF}}(k)\right)\right)$ were also considered.

Finally, another spectral parameter, named peakness $(\wp)$, was computed. It was already used in a previous work for the characterization of a population of children classified attending to their risk of developing asthma [20]. Basically, it accounts for the distribution of the HF components of HRV around the respiratory rate. Thus, $\wp$ is defined as:

$$
\wp(k)=\frac{\int_{\max \left(\overline{F_{\mathrm{r}}}(k)-\Delta f / 2,0.15\right)}^{\min \left(\overline{F_{\mathrm{r}}}(k)+\Delta f / \overline{\mathrm{HR}}(k) / 2\right)} \hat{S}_{\mathrm{HRV}}(k, F) d F}{\int_{\max \left(\overline{F_{\mathrm{r}}}(k)-\Delta F / 2,0.15\right)}^{\min (\overline{\mathrm{HR}}(k) / 2)} \hat{S}_{\mathrm{HRV}}(k, F) d F}
$$

where $\Delta f$ is $0.026 \mathrm{~Hz}$ (resolution of the Hamming window), $\Delta F$ is the $0.15 \mathrm{~Hz}$ frequency window employed in the definition of the $\mathrm{HF}$ band, and $\overline{F_{\mathrm{r}}}(k)$ and $\overline{\mathrm{HR}}(k)$ are the mean $F_{\mathrm{r}}$ and $\mathrm{HR}$ in the $k$-th five-minute window respectively (the upper limit of $\overline{\mathrm{HR}}(k) / 2$ was used since HR remains the intrinsic sampling frequency of HRV [38]). In this way, the value of $\wp$ will be closer to 1 as the HF components are more concentrated around $F_{\mathrm{r}}$.

\section{Time-Frequency Coherence Analysis}

The time-frequency (TF) cross-spectrum of the HRV and the IP signals, $\hat{S}_{\text {HRV,IP }}(t, f)$, was estimated using a TF distribution belonging to Cohen's class, defined as in [39]:

$$
\hat{S}_{\mathrm{HR}, \mathrm{IP}}(t, f)=\iint_{-\infty}^{\infty} A_{\mathrm{HRV}, \mathrm{IP}}(\nu, \tau) \Phi(\nu, \tau) e^{j 2 \pi(t \nu-f \tau)} d \nu d \tau,
$$

where $A_{\mathrm{HRV}, \mathrm{P}}(\nu, \tau)$ is the ambiguity function [40] of the analytical signal representation of the modulating and the IP signals, $x_{\mathrm{HRV}}(t)$ and $x_{\mathrm{IP}}(t)$, obtained using the Hilbert transform. On the other hand, $\Phi(\nu, \tau)$ is a smoothing function in the ambiguity domain (in this work it was selected as an elliptic exponential kernel). They are respectively defined as:

$$
\begin{gathered}
A_{\mathrm{HRV}, \mathrm{IP}}(\nu, \tau)=\int_{-\infty}^{\infty} x_{\mathrm{HRV}}\left(t+\frac{\tau}{2}\right) x_{\mathrm{IP}}^{*}\left(t-\frac{\tau}{2}\right) e^{-j 2 \pi \nu t} d t, \\
\Phi(\nu, \tau)=e^{-\pi\left[\left(\frac{\nu}{\nu_{0}}\right)^{2}+\left(\frac{\tau}{\tau_{0}}\right)^{2}\right]^{2 \lambda}} .
\end{gathered}
$$

The time and frequency resolution of $\hat{S}_{\mathrm{HR}, \mathrm{IP}}(t, f)$ can be adjusted by modifying the shape of the smoothing kernel in Eq. 5 through the parameters $\nu_{0}$ and $\tau_{0}$, respectively. Also the roll-off factor of the kernel can be controlled with $\lambda$. In this work, these parameters were fixed to $\nu_{0}=0.045, \tau_{0}=0.05$ and $\lambda=0.3$, as in [41], yielding to a time and frequency

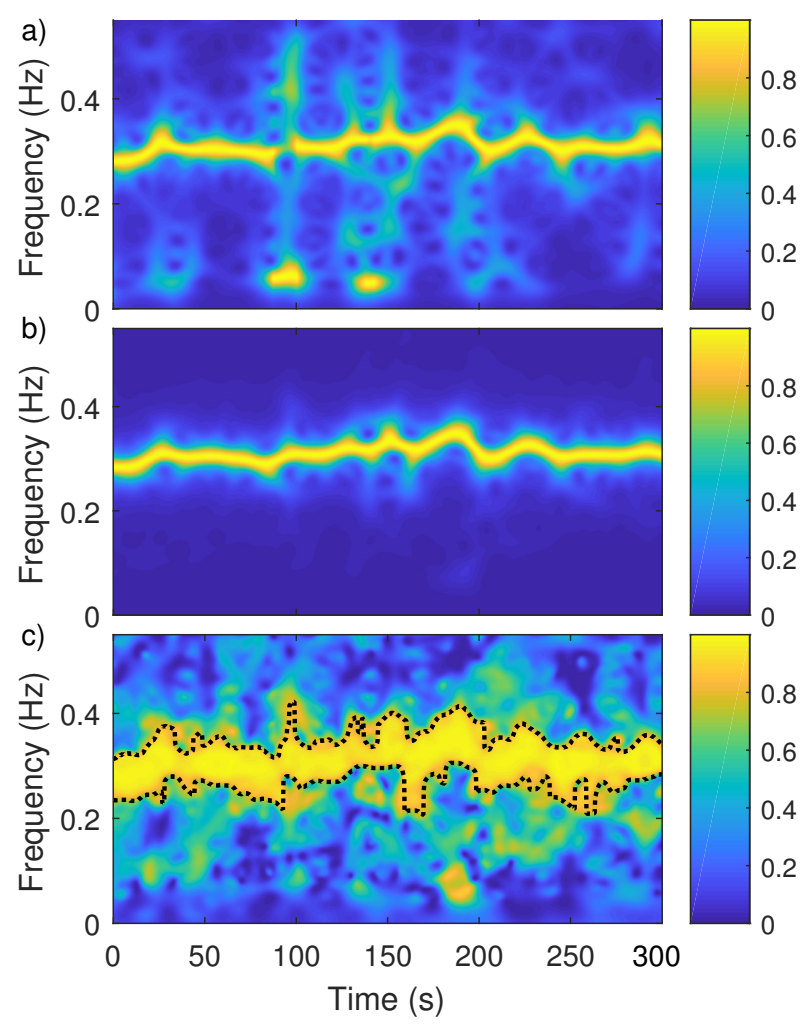

Fig. 2. A five-minute segment of the normalized time-frequency distribution of the heart rate modulating signal (a)) and the impedance pneumography signal (b)) are displayed. In c), the time-frequency coherence distribution is depicted. The black dotted lines represent the limits between which $\hat{\gamma}^{2}(t, f) \geq \gamma_{\mathrm{TH}}(t, f ; \alpha)$, defining $\Omega(t)$ (see text for details).

resolution of $11.25 \mathrm{~s}$ and $0.039 \mathrm{~Hz}$ respectively (time and frequency resolution were calculated as the full width at half maximum of the TF distribution of a Dirac impulse evaluated along $t$, and of a pure sinusoid evaluated along $f$ ). Afterwards, the TF coherence (TFC) distribution was obtained as:

$$
\hat{\gamma}^{2}(t, f)=\frac{\left|\hat{S}_{\mathrm{HRV}, \mathrm{IP}}(t, f)\right|^{2}}{\hat{S}_{\mathrm{HRV}}(t, f) \hat{S}_{\mathrm{IP}}(t, f)},
$$

where $\hat{S}_{\mathrm{HRV}}(t, f)$ and $\hat{S}_{\mathrm{IP}}(t, f)$ represent the TF spectra of the modulating and the IP signals respectively, estimated as in Eq. 4. Finally, two different parameters were proposed as CRC measurements. The first one consists in the bandwidth $\Omega(t)$, in which both spectra are considered coherent for each time instant $t$, i.e., the bandwidth for which $\hat{\gamma}^{2}(t, f) \geq \gamma_{\text {тн }}(t, f ; \alpha)$, being $\gamma_{\text {тн }}(t, f ; \alpha)$ the signal-independent threshold defined in [39], with $\alpha=0.01$. An example of the calculation of $\Omega$ is displayed in Fig. 2. Moreover, the mean coherence in $\Omega(t)$, $\hat{\gamma}_{\Omega}^{2}(t)$, was obtained as:

$$
\hat{\gamma}_{\Omega}^{2}(t)=\frac{1}{\Omega(t)} \int_{\Omega_{1}(t)}^{\Omega_{2}(t)} \hat{\gamma}^{2}(t, f) d f
$$

being $\Omega_{1}(t)$ and $\Omega_{2}(t)$ the lower and upper limits of the frequency band for which $\hat{\gamma}^{2}(t, f) \geq \gamma_{\mathrm{TH}}(t, f ; \alpha)$ in the time instant $t$. 


\section{E. Statistical analysis}

Median of each parameter was obtained from two-hour windows, so that at least one complete sleep cycle is covered [42] and the effect of sleep stages is hence minimized. A twohour median was calculated every hour from 23:00 p.m. to 06:00 a.m., thus resulting in 8 windows per parameter, subject and recording day. Afterwards, the subjects were analyzed attending to the three different classification criteria (CA status, atopy and response to treatment). For each of these criteria, each two-hour median of each subject was compared along the three recording days, using a Friedman test and the Dunn-Sidak's criteria for multiple comparisons. On the other hand, in order to assess if the presence of atopy or the response to medication had an effect in the results obtained for the classification according to the CA status, differences among these subgroups within the CA-N group were assessed using a Wilcoxon rank-sum test. In all the tests, $\mathrm{p}<0.05$ was set as the significance level to consider statistical differences.

\section{RESUltS}

Since children were labeled attending to their current asthma statuts, atopy and response to ICS treatment, the results of the analysis were evaluated considering each of these classifications independently. The greatest amount of significant differences was obtained for the HRV indexes accounting for parasympathetic activity and the CRC parameters so, for simplicity, only they were considered below. From all the available subjects, there were 3 with the 3 recordings absent or discarded due to bad quality, and also 10 for which 2 out of 3 recordings were not considered for the same reasons. Since inter-day behavior of the different parameters followed a similar tendency along the night, results obtained at the hour with the greatest amount of significant differences, i.e., at 03 a.m., are summarized in Tables I, II and III. Additionally, boxplots of the two-hours median of some of the considered parameters and an example of the time course of the most relevant one are displayed in Figs. 3 and 4, respectively.

Attending to current asthma status: A clear tendency towards increased $\mathrm{P}_{\mathrm{LFn}}$ and decreased RMSSD, $\mathrm{P}_{\mathrm{HF}}$ and $\wp$ was found in R3 with respect to R1 and R2 in CA-N. This behavior was consistent along several parameters and twohour windows, as displayed in Fig. 3. These differences were specially noticeable at 03 and 04 a.m. in the frequency-domain HRV indexes. A similar behavior was assessed in the CRC parameters (see Fig. 3). On the other hand, no differences between the three recording days were found in CA-Y and CA-P.

Attending to atopic status: Patients classified as atopic presented significantly decreased $\wp$ in R 3 with respect to R2 at 03 a.m. In the case of non-atopic subjects, they presented a tendency towards decreased $\Omega$ and $\hat{\gamma}_{\Omega}^{2}$ in R3 with respect to R2 and R1, which turned statistically significant for $\Omega$ at 01 a.m. No differences were assessed in any of the other considered parameters for none of the groups.

Attending to the response to treatment: A tendency towards decreased $\overline{\mathrm{NN}}$, RMSSD and $\mathrm{P}_{\mathrm{HF}}$, and incresed $\mathrm{P}_{\mathrm{LFn}}$ was assessed in the group who did not responded to treatment, being them significant from 04 to 05 a.m. Regarding the CRC indexes, decreased $\Omega$ and $\hat{\gamma}_{\Omega}^{2}$ were assessed in the group with partial response to treatment from 01 to 02 and at 02 a.m. respectively. The same behavior was found at 00 a.m. in the group which responded effectively to treatment.

Finally, when comparing the different atopy or response to treatment groups within the CA-N group, no differences were assessed for any tow-hours window or recording day.

\section{DISCUSSION}

All the children underwent the same ICS treatment during three months. However, whereas ANS activity (as measured from HRV) remained unchanged after treatment completion in those children classified as CA-Y, a decrease in parasympathetic activity and an increase in sympathetic dominance was observed in the CA-N group. Since previous studies have assessed an augmented vagal activity in asthmatics [15], [18], [19], the lowered PNS activity in CA-N following treatment could be reflecting recovery from illness, so that vagal overactivity is gradually diminished towards homeostatic levels. Also a reduction in $\wp$ and CRC (as meadured from $\Omega$ and $\hat{\gamma}_{\Omega}^{2}$ ) were assessed in this group. Since CRC is related to how the respiratory activity modulates the HR, a reduction in CRC measurements and $\wp$ could be reflecting a less synchronous vagal modulation of respiratory and cardiac rhythms or a less regular PNS activity, respectively. In this case, the interpretation might be linked to the concept of illness as a state of reduced complexity [43], [44], suggesting that HRV might be more dependent on the respiratory activity in asthma or in subjects at an increased risk of developing asthma in the future. The fact that both $\Omega$ and $\hat{\gamma}_{\Omega}^{2}$ were lowered in R3 in the CA-N group suggests a reduction in the frequency span in which HRV is governed by respiration, but also in the strength of the interdependence of cardiac and respiratory control in those subjects that have overcome the disease. This hypothesis is supported by previous studies suggesting lowered chaoticity and regularity of impedance pneumography [45] and airflow pattern [46] in subjects with a worse asthma outcome. It is noteworthy that, in concordance with our previous work [20], most significant differences were obtained between 02 and 04 a.m., thus when airway function is lowest [26], [47].

Despite not being specific for asthma, the presence of atopy enhances the probability that a patient with respiratory symptoms presents allergic asthma [1], and it has been included as a factor for the prediction of asthma in, e.g., the Isle of Wight study [48]. For these reasons, we analyzed the results regarding the atopic status of the patients. However, the absence of differences regarding the atopic status in all the considered indexes suggests that it is not closely related with the apparently altered ANS activity in CA-Y.

On the other hand, whereas changes in ANS function were expected to be produced by a proper response to ICS treatment, no differences were found in the HRV parameters of the group for which treatment resulted effective (which constitutes the majority of the dataset). However, small differences were found in the group with partial response to treatment, specially in the CRC. The most likely explanation for this outcome is 
TABLE I

MEDIAN AND $\left[25^{t h}, 75^{t h}\right.$ PERCENTILES OF THE MOST RELEVANT TIME DOMAIN HEART RATE VARIABILITY PARAMETERS OBTAINED FROM A TWO-HOUR WINDOW CENTERED AT 03 A.M. RESULTS FOR EACH RECORDING DAY ATTENDING TO THEIR CURRENT ASTHMA STATUS, ATOPY AND RESPONSE TO TREATMENT ARE DISPLAYED. STATISTICAL SIGNIFICANT DIFFERENCES WITH R1 ARE INDICATED WITH * (P $\leq 0.05)$, WHEREAS DIFFERENCES WITH R2 ARE LABELED WITH $\dagger(\mathrm{P} \leq 0.05)$.

\begin{tabular}{|c|c|c|c|c|c|c|}
\hline & \multicolumn{3}{|c|}{$\overline{\mathrm{NN}}(\mathrm{ms})$} & \multicolumn{3}{|c|}{ RMSSD (ms) } \\
\hline & R1 & R2 & R3 & $\mathrm{R} 1$ & R2 & R3 \\
\hline \multicolumn{7}{|l|}{ Attending to asthma: } \\
\hline - $\mathrm{CA}-\mathrm{N}$ & $718.6[676.5,811.3]$ & $693.9[645.3,772.8]$ & $686.1[639.4,725.9]$ & $69.36[32.01,136.12]$ & $58.24[36.32,98.65]$ & $45.13[27.67,98.91]$ \\
\hline - CA-P & $718.6[693.0,817.7]$ & 709.6 [641.6, 819.4] & $731.8[675.5,799.2]$ & $70.37[57.11,120.54]$ & $62.26[28.27,96.53]$ & $83.90[32.60,136.66]$ \\
\hline - $\mathrm{CA}-\mathrm{Y}$ & $712.1[637.3,773.2]$ & $708.8[629.6,774.3]$ & $713.8[663.2,768.2]$ & $71.16[49.35,122.54]$ & $83.08[48.15,128.86]$ & $74.22[44.22,119.90]$ \\
\hline \multicolumn{7}{|l|}{ Attending to SPT: } \\
\hline - Non-atopic & $703.0[668.4,735.2]$ & $693.2[624.4,752.2]$ & $701.5[640.3,736.8]$ & $67.59[44.64,104.49]$ & $57.01[39.19,98.07]$ & $57.76[30.96,95.68]$ \\
\hline - Atopic & $773.9[667.9,849.6]$ & $737.7[662.1,832.7]$ & $713.8[672.4,828.2]$ & $94.60[57.28,132.02]$ & $85.30[44.97,119.47]$ & $89.57[45.37,121.23]$ \\
\hline \multicolumn{7}{|l|}{ Attending to treatment: } \\
\hline - No effective & $703.2[680.1,840.5]$ & $673.4[632.8,711.5]^{*}$ & $701.5[626.0,738.6]$ & $70.37[63.01,150.73]$ & $51.15[34.12,93.40]$ & $57.95[31.02,93.08]^{*}$ \\
\hline - Partially effective & $705.4[637.3,730.4]$ & $669.7[640.8,772.4]$ & $680.2[639.3,715.8]$ & $48.97[27.58,76.96]$ & $46.39[30.96,96.33]$ & $45.13[23.10,75.48]$ \\
\hline - Effective & $730.5[677.6,815.8]$ & $718.7[634.3,781.1]$ & $721.9[672.4,790.3]$ & $76.78[52.93,126.18]$ & $80.14[49.89,118.71]$ & $84.77[43.07,121.23]$ \\
\hline
\end{tabular}

TABLE II

MEDIAN AND $\left[25^{t h}, 75^{t h}\right.$ ] PERCENTILES OF THE MOST RELEVANT FREQUENCY DOMAIN HEART RATE VARIABILITY PARAMETERS OBTAINED FROM A TWO-HOUR WINDOW CENTERED AT 03 A.M. RESULTS FOR EACH RECORDING DAY ATTENDING TO THEIR CURRENT ASTHMA STATUS, ATOPY AND RESPONSE TO TREATMENT ARE DISPLAYED. STATISTICAL SIGNIFICANT DIFFERENCES WITH R1 ARE INDICATED WITH * (P $\leq 0.05)$, wHEREAS DIFFERENCES WITH R2 ARE LABELED WITH $\dagger(\mathrm{P} \leq 0.05)$.

\begin{tabular}{|c|c|c|c|c|c|c|c|c|c|}
\hline & \multicolumn{3}{|c|}{$\mathrm{P}_{\mathrm{HF}}\left(\mathrm{ad} \times 10^{-3}\right)$} & \multicolumn{3}{|c|}{$\mathrm{P}_{\mathrm{LFn}}$ (n.u.) } & \multicolumn{3}{|c|}{$\wp$ (n.u.) } \\
\hline & R1 & R2 & R3 & R1 & R2 & R3 & $\mathrm{R} 1$ & R2 & R3 \\
\hline \multicolumn{10}{|l|}{ Attending to asthma: } \\
\hline - $\mathrm{CA}-\mathrm{N}$ & $3.28[0.99,8.02]$ & $3.28[1.53,5.84]$ & $1.78[0.59,5.23]^{*}$ & $0.35[0.27,0.45]$ & $0.35[0.31,0.43]$ & $0.48[0.36,0.53]^{*}$ & $0.37[0.31,0.41]$ & $0.41[0.34,0.47]$ & $0.34[0.28,0.39]^{\dagger}$ \\
\hline - CA-P & $3.56[2.12,7.76]$ & $2.36[0.65,5.30]$ & $3.69[0.96,12.47]$ & $0.37[0.26,0.46$ & $0.48[0.25,0.56]$ & $0.41[0.29,0.46]$ & $0.39[0.32,0.42]$ & $0.37[0.34,0.43]$ & $0.40[0.32,0.43]$ \\
\hline - $\mathrm{CA}-\mathrm{Y}$ & $3.43[1.16,7.45]$ & $3.80[1.57,8.62]$ & $3.57[1.19,8.48]$ & $0.32[0.25,0.53]$ & $0.33[0.26,0.41]$ & $0.33[0.28,0.47]$ & $0.38[0.32,0.47]$ & $0.38[0.34,0.42]$ & $0.38[0.35,0.44]$ \\
\hline \multicolumn{10}{|l|}{ Attending to SPT: } \\
\hline - Non-atopic & $2.63[1.15,6.47]$ & $2.27[1.33,5.71]$ & $2.21[0.66,5.23]$ & $0.35[0.29,0.54]$ & $0.38[0.29,0.54]$ & $0.43[0.34,0.53]$ & $0.39[0.30,0.45]$ & $0.38[0.31,0.46]$ & $0.36[0.30,0.46]$ \\
\hline - Atopic & $4.32[2.25,8.47]$ & $4.88[1.86,7.08]$ & $4.61[1.72,9.02]$ & $0.28[0.23,0.42]$ & $0.33[0.23,0.43]$ & $0.32[0.28,0.43]$ & $0.36[0.31,0.43]$ & $0.42[0.37,0.46]$ & $0.38[0.34,0.42]^{\dagger}$ \\
\hline \multicolumn{10}{|l|}{ Attending to treatment: } \\
\hline - No effective & $3.56[2.22,8.51]$ & $2.10[1.19,4.39]$ & $2.40[0.79,3.98]$ & $0.36[0.26,0.41]$ & $0.38[0.36,0.58]$ & $0.47[0.41,0.50]$ & $0.33[0.32,0.42]$ & $0.35[0.30,0.39]$ & $0.36[0.26,0.38]$ \\
\hline - Partially effective & $2.50[0.68,5.70]$ & $2.14[0.96,7.37]$ & $1.72[0.58,4.04]$ & $0.35[0.25,0.62]$ & $0.35[0.25,0.44]$ & $0.45[0.34,0.55]$ & $0.37[0.31,0.44]$ & $0.41[0.33,0.50]$ & $0.36[0.32,0.44]$ \\
\hline - Effective & $4.06[1.89,8.01]$ & $3.78[1.59,6.81]$ & $4.19[1.12,9.02]$ & $0.33[0.26,0.50]$ & $0.34[0.25,0.47]$ & $0.36[0.28,0.46]$ & $0.39[0.31,0.45]$ & $0.41[0.33,0.45]$ & $0.38[0.33,0.44]$ \\
\hline
\end{tabular}

TABLE III

MEDIAN AND $\left[25^{t h}, 75^{t h}\right.$ ] PERCENTILES OF THE PROPOSED CARDIORESPIRATORY COUPLING PARAMETERS OBTAINED FROM A TWO-HOUR WINDOW CENTERED AT 03 A.M. RESULTS FOR EACH RECORDING DAY ATTENDING TO THEIR CURRENT ASTHMA STATUS, ATOPY AND RESPONSE TO TREATMENT ARE DISPLAYED. STATISTICAL SIGNIFICANT DIFFERENCES WITH R1 ARE INDICATED WITH * (P $\leq 0.05)$, WHEREAS DIFFERENCES WITH R2 ARE LABELED WITH $\dagger(\mathrm{P} \leq 0.05)$.

\begin{tabular}{|c|c|c|c|c|c|c|}
\hline & \multicolumn{3}{|c|}{$\begin{array}{l}\Omega(\mathrm{Hz}) \\
\end{array}$} & \multicolumn{3}{|c|}{$\hat{\gamma}_{\Omega}^{2}$ (n.u) } \\
\hline & $\mathrm{R} 1$ & R2 & R3 & R1 & R2 & R3 \\
\hline \multicolumn{7}{|l|}{ Attending to asthma: } \\
\hline - CA-N & $0.089[0.074,0.105]$ & $0.086[0.078,0.111]$ & $0.083[0.073,0.097]^{*, \dagger}$ & $0.911[0.901,0.917]$ & $0.911[0.908,0.919]$ & $0.910[0.903,0.913]^{\dagger}$ \\
\hline - CA-P & $0.101[0.080,0.111]$ & $0.088[0.077,0.106]$ & $0.093[0.077,0.107]$ & $0.916[0.907,0.919]$ & $0.912[0.903,0.914]$ & $0.911[0.905,0.918]$ \\
\hline - CA-Y & $0.090[0.075,0.101]$ & $0.092[0.082,0.104]$ & $0.088[0.076,0.105]$ & $0.913[0.906,0.917]$ & $0.914[0.908,0.917]$ & $0.912[0.906,0.917]$ \\
\hline \multicolumn{7}{|l|}{ Attending to SPT: } \\
\hline - Non-atopic & $0.086[0.076,0.104]$ & $0.086[0.076,0.106]$ & $0.087[0.073,0.100]$ & $0.913[0.904,0.917]$ & $0.911[0.907,0.916]$ & $0.910[0.903,0.917]$ \\
\hline - Atopic & $0.089[0.076,0.104]$ & $0.091[0.084,0.108]$ & $0.090[0.076,0.105]$ & $0.913[0.906,0.918]$ & $0.915[0.908,0.917]$ & $0.913[0.905,0.917]$ \\
\hline \multicolumn{7}{|l|}{ Attending to treatment: } \\
\hline - No effective & $0.086[0.075,0.109]$ & $0.076[0.074,0.100]$ & $0.089[0.074,0.105]$ & $0.913[0.902,0.917]$ & $0.905[0.902,0.915]$ & $0.911[0.903,0.916]$ \\
\hline - Partially effective & $0.089[0.076,0.105]$ & $0.086[0.079,0.110]$ & $0.083[0.072,0.103]$ & $0.911[0.905,0.918]$ & $0.912[0.908,0.917]$ & $0.910[0.904,0.917]$ \\
\hline - Effective & $0.090[0.076,0.108]$ & $0.091[0.082,0.107]$ & $0.088[0.076,0.101]$ & $0.913[0.906,0.918]$ & $0.913[0.907,0.917]$ & $0.912[0.906,0.917]$ \\
\hline
\end{tabular}

that most of the children for which the treatment was effective were classified as CA-Y or CA-P, whereas the group with partial response to ICS is mainly formed by subjects classified as CA-N (Fig. 1), thus suggesting that the observed differences may not be related with the treatment but with the evolution of the illness itself. This result is specially interesting, as it could indicate that, despite ICS treatment which is aimed to reduce airway inflammation, altered ANS behavior might be still present in the groups with a worse asthma prognosis.

Nevertheless, the fact that children with different atopic or response to treatment status are classified as CA-N could have a direct influence in the observed results. In this way, we studied the differences between atopics and non-atopics classified as CA-N, as well as between the subgroups attending to the response to treatment. The absence of significant differences suggests that the observed results attending to the CA status are not likely due to the atopic condition or the response to treatment of the different subjects.

The main limitation of this work resides in the absence of polysomnographic recordings, so that it was not possible to look for differences in HRV along the various sleep stages. In order to deal with this restriction, we proposed to calculate 
CA-N
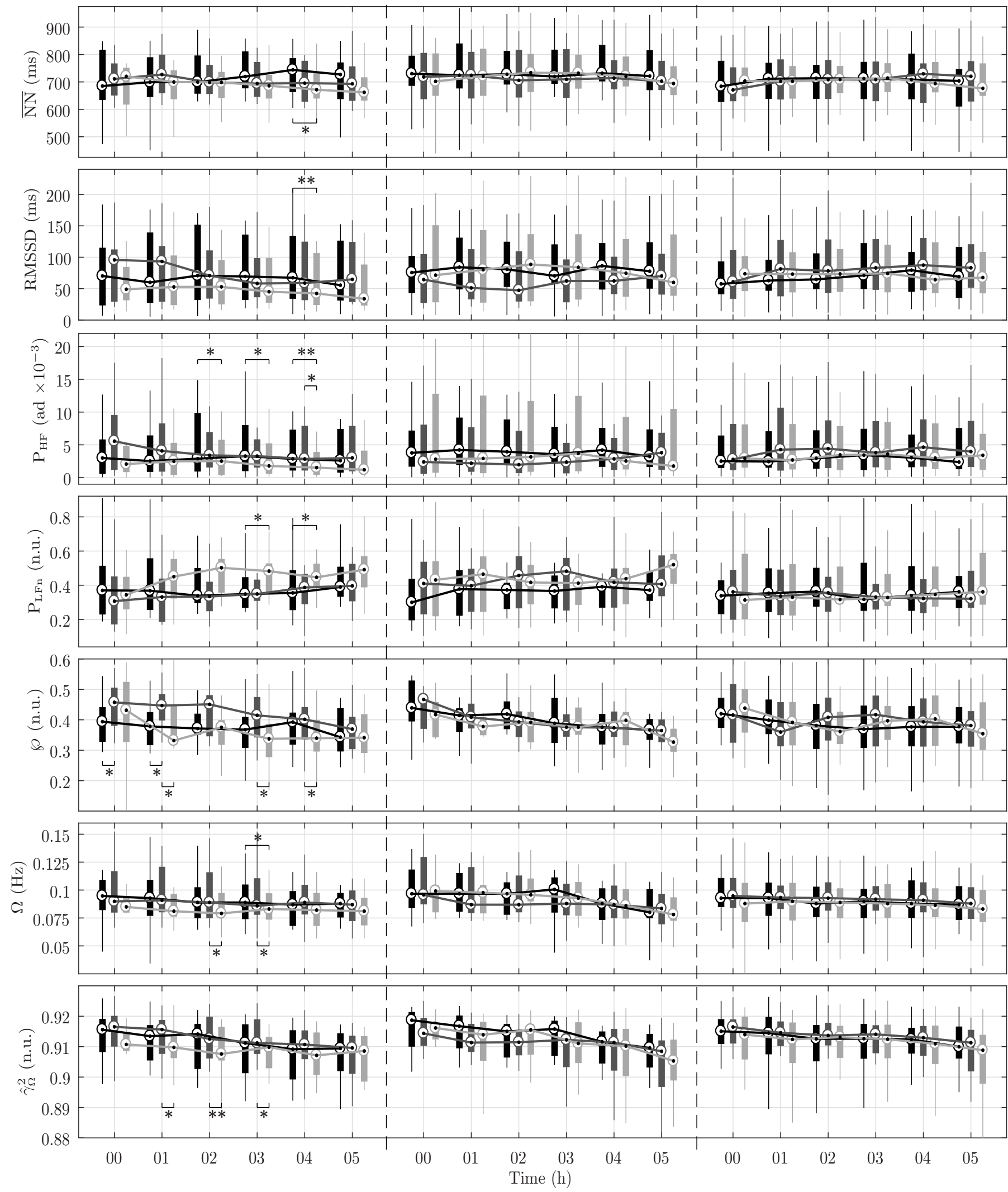

Fig. 3. Boxplots corresponding to some of the analyzed parameters in R1 (black), R2 (dark gray) and R3 (light gray) attending to the current asthma status. Each box corresponds to a two-hour window centered in the hour depicted in the figure (although boxes with the same time reference are depicted separately for clarity, same central hour was considered in the analysis). Medians of the boxes corresponding to the same measurement day are connected with solid lines, and statistical significant differences are labeled with $*(\mathrm{p} \leq 0.05)$ or $* *(\mathrm{p} \leq 0.005)$. $\mathrm{P}_{\mathrm{HF}}$, as obtained from $m(n)$, is adimensional (ad). For representation purposes, the results corresponding to the two-hour windows centered at 23 p.m. and 06 a.m. are not depicted (no differences were assessed in these cases). 

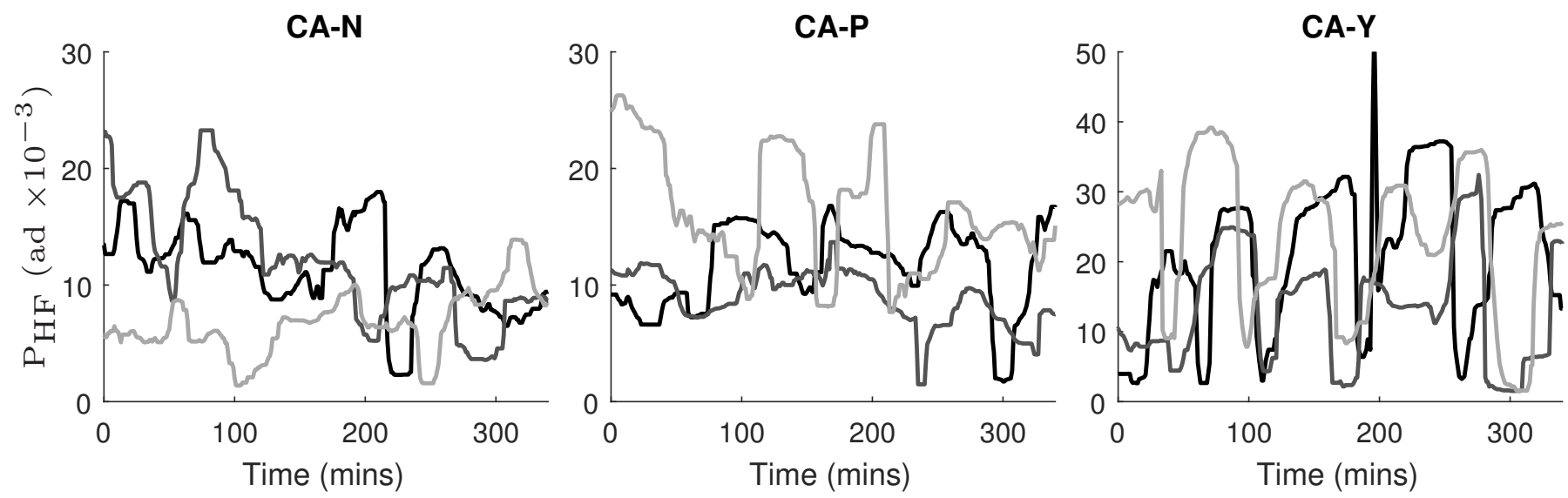

Fig. 4. Time course of $\mathrm{P}_{\mathrm{HF}}$ for R1 (black), R2 (dark gray) and R3 (light gray) of three subjects belonging to the different current asthma groups. $\mathrm{P}_{\mathrm{HF}}$, as obtained from $m(n)$, is adimensional (ad).

two-hour medians of the analyzed indexes to cover at least one complete sleep cycle [42]. Furthermore, we checked that the overnight variation was not higher than the interday variation for any of the considered parameters, being $\overline{\mathrm{NN}}$, RMSSD and $\mathrm{P}_{\mathrm{HF}}$ those that showed a larger inter-day variability (results not shown). The fact that respiratory rate is higher in children than in adults can also compromise the traditional HRV analysis, given that the upper bound of the HF band results insufficient for an accurate measurement of the HF power. In this way, the $\mathrm{HF}$ band was redefined adaptively in function of the respiratory rate as already done in [20]. Other works have emphasized the need of redefining the HF band for HRV analysis in children [49], although we opted for a respiratory-guided band instead of a fixed one for two reasons: first, since the children are sleeping, the HF content is concentrated around the respiratory rate. Second, by using a band centered in respiration, we can avoid the inclusion of alias or frequency components with an uncertain origin [50]. Also the methodology for dealing with the stronger effect of RSA in children employed here was introduced in [20], where the threshold for distinguishing between RSA and ectopic beats was set to 1.15, following a previous work in which they employed this threshold for detecting premature ventricular contractions [51]. However, in the current study that threshold resulted insufficient for capturing all the RSA episodes, so we adapted it to the considered dataset, setting it to 1.5 . The possibility of using a unique threshold which is independent of the data remains to be investigated.

In spite of the many studies stating the important role of PNS as a source of the airway hyper-responsiveness characteristic of asthma, the underlying mechanisms causing an abnormal vagal activity have not been elucidated. The presence of immune cells which are involved in the inflammatory response has been considered as one likely explanation, as they release inflammatory mediators that could alter local PNS activity and trigger bronchial hyper-reactivity. However, several counterpoints can be highlighted. First, preventive treatment with antiinflammatory corticosteroids has been shown to be insufficient for avoiding the development of asthma in young children [52]. Second, the presence of different phenotypes of asthma with distinct manifestations and responses to treatment, together with an apparent absence of relationship between inflammation and airway remodeling in children [53] suggest the existence of other factors. Moreover, the fact that altered PNS activity in asthmatics can be also noticed in HRV opposes to the idea of a local effect. Fryer et al. suggested that excessive vagal stimulation could be caused by a dysfunction of the $M_{2}$ muscarinic receptors [54], which are largely present in the post-ganglionic nerves innervating the airways and provide negative feedback in response to acetylcholine (ACh) withdrawal, thus inhibiting the further release of ACh. Although the lowered control over $\mathrm{ACh}$ release might explain the increased vagal dominance observed through HRV in the presence of asthma, as well as the more regular PNS activity suggested by increased $\wp$, if cardiac vagal fibers also presented $M_{2}$ receptors dysfunction, the simultaneous reduction in the adaptability of cardiac and respiratory vagal control due to excessive ACh release could resemble an increased CRC. In this way, further research is needed to completely understand the neural control in asthmatics.

\section{CONCLuSion}

HRV analysis has been presented as a suitable noninvasive tool for the assessment of abnormal ANS activity in children at risk of asthma. The main outcome of this work is that vagal activity and cardiorespiratory coupling, measured in a group of children with obstructive bronchitis, were reduced after ICS treatment in the subgroup at lower risk of asthma, whereas it kept unchanged in those who presented a worse prognosis. The difficulties of young children to perform repeatable spirometric tests together with the lack of collaboration and the low adherence to ICS treatment emphasize the interest in a continuous monitoring of asthma in order to detect or predict exacerbations, thus providing an objective measurement of the evolution of the disease. In this way, HRV stands out as a feasible option. In addition, the possibility of a continuous monitoring of ANS activity in asthmatics could shed some light on the physiological mechanisms underlying asthma, and hence be useful for patient phenotyping, which constitutes the first step towards personalized treatment. 


\section{REFERENCES}

[1] Global Initiative for Asthma. Global strategy for asthma management and prevention, 2018. Available from www.ginasthma.org.

[2] H. Eigen, et al., "Spirometric pulmonary function in healthy preschool children," Am J Respir Crit Care Med, vol. 163, no. 3, pp. 619-623, 2001.

[3] N. Beydon, et al., "An official american thoracic society/european respiratory society statement: pulmonary function testing in preschool children," Am J Respir Crit Care Med, vol. 175, no. 12, pp. 1304-1345, 2007.

[4] C. Pesant, et al., "Spirometric pulmonary function in 3-to 5-year-old children," Pediatr Pulmonol, vol. 42, no. 3, pp. 263-271, 2007.

[5] S. Young, et al., "The association between early life lung function and wheezing during the first 2 yrs of life," Eur Respir J, vol. 15, no. 1, pp. 151-157, 2000.

[6] S. Lum, et al., "Early detection of cystic fibrosis lung disease: multiplebreath washout vs. raised volume tests," Thorax, 2006.

[7] S. Lum, et al., "Potential misinterpretation of infant lung function unless prospective healthy controls are studied," Pediatr Pulmonol, vol. 45, no. 9, pp. 906-913, 2010.

[8] E. Heffler, et al., "Inhaled corticosteroids safety and adverse effects in patients with asthma," J Allergy Clin Immunol Pract, vol. 6, no. 3, pp. 776-781, 2018.

[9] O. Cavkaytar, et al., "Evidence of hypothalamic-pituitary-adrenal axis suppression during moderate-to-high-dose inhaled corticosteroid use," Eur J Pediatr, vol. 174, no. 11, pp. 1421-1431, 2015.

[10] R. Dahl, "Systemic side effects of inhaled corticosteroids in patients with asthma," Respir Med, vol. 100, no. 8, pp. 1307-1317, 2006.

[11] L.-P. Boulet, et al., "Adherence: the goal to control asthma," Clin Chest Med, vol. 33, no. 3, pp. 405-417, 2012.

[12] S. Stanojevic, A. Wade, and J. Stocks, "Reference values for lung function: past, present and future," Eur Respir J, vol. 36, no. 1, pp. 12 $19,2010$.

[13] L. Agertoft and S. Pedersen, "Effects of long-term treatment with an inhaled corticosteroid on growth and pulmonary function in asthmatic children," Respir Med, vol. 88, no. 5, pp. 373-381, 1994.

[14] M. Kaliner, et al., "Autonomic nervous system abnormalities and allergy," Ann Intern Med, vol. 96, no. 3, pp. 349-357, 1982.

[15] O. Emin, et al., "Autonomic nervous system dysfunction and their relationship with disease severity in children with atopic asthma," Respir Physiol Neurobiol, vol. 183, no. 3, pp. 206-210, 2012.

[16] M. Lewis, A. Short, and K. Lewis, "Autonomic nervous system control of the cardiovascular and respiratory systems in asthma," Respir Med, vol. 100, no. 10, pp. 1688-1705, 2006.

[17] J. Morrison, S. Pearson, and H. Dean, "Parasympathetic nervous system in nocturnal asthma," $\mathrm{Br}$ Med J (Clin Res Ed), vol. 296, no. 6634, p. $1427,1988$.

[18] J. Kallenbach, et al., "Reflex heart rate control in asthma: evidence of parasympathetic overactivity," Chest, vol. 87, no. 5, pp. 644-648, 1985.

[19] B. Zahorska-Markiewicz, et al., "Circadian heart rate variability in asthma," Med Sci Monit, vol. 3, no. 1, pp. CR52-CR56, 1997.

[20] J. Milagro, et al., "Nocturnal heart rate variability spectrum characterization in preschool children with asthmatic symptoms," IEEE J Biomed Health Inform, vol. 22, no. 5, pp. 1332-1340, 2018.

[21] A. J. Garcia III, et al., "Cardiorespiratory coupling in health and disease," Auton Neurosci, vol. 175, no. 1-2, pp. 26-37, 2013.

[22] J. Milagro, et al., "Nonlinear dynamics of heart rate variability in children with asthmatic symptoms," in EMBEC \& NBC 2017, pp. 815818, Springer, 2017.

[23] V.-P. Seppä, et al., "Tidal breathing flow measurement in awake young children by using impedance pneumography," J App Physiol, vol. 115, no. 11 , pp. 1725-1731, 2013.

[24] L. P. Malmberg, et al., "Measurement of tidal breathing flows in infants using impedance pneumography," Eur Resp J, vol. 49, no. 2, pp. 1600926, 2017

[25] P. Bušek, et al., "Spectral analysis of heart rate variability in sleep," Physiol Res, vol. 54, no. 4, pp. 369-376, 2005.

[26] P. J. Barnes, "Circadian variation in airway function," Am J Med, vol. 79, no. 6, pp. 5-9, 1985.

[27] R. D. Ballard, et al., "Effect of sleep on nocturnal bronchoconstriction and ventilatory patterns in asthmatics," J Appl Physiol, vol. 67, no. 1, pp. 243-249, 1989.

[28] M. Merri, et al., "Sampling frequency of the electrocardiogram for spectral analysis of the heart rate variability," IEEE Trans Biomed Eng, vol. 37 , no. 1 , pp. 99-106, 1990.
[29] J. P. Martínez, et al., "A wavelet-based ECG delineator: evaluation on standard databases," IEEE Trans Biomed Eng, vol. 51, no. 4, pp. 570$581,2004$.

[30] J. Mateo and P. Laguna, "Analysis of heart rate variability in the presence of ectopic beats using the heart timing signal," IEEE Trans Biomed Eng, vol. 50, no. 3, pp. 334-343, 2003.

[31] M. Nagashima, et al., "Cardiac arrhythmias in healthy children revealed by 24-hour ambulatory ecg monitoring," Pediatr Cardiol, vol. 8, no. 2, pp. 103-108, 1987.

[32] K. Niwa, et al., "Prevalence of arrhythmias and conduction disturbances in large population-based samples of children," Cardiol Young, vol. 14, no. 1, pp. 68-74, 2004.

[33] J. P. Finley and S. T. Nugent, "Heart rate variability in infants, children and young adults," J Auton Nerv Syst, vol. 51, no. 2, pp. 103-108, 1995.

[34] J. Lázaro, et al., "Electrocardiogram derived respiratory rate from QRS slopes and R-wave angle," Ann Biomed Eng, vol. 42, no. 10, pp. 20722083, 2014.

[35] R. Bailón, L. Sornmo, and P. Laguna, "A robust method for ECG-based estimation of the respiratory frequency during stress testing," IEEE Trans Biomed Eng, vol. 53, no. 7, pp. 1273-1285, 2006.

[36] Task Force of the European Society of Cardiology, "Heart rate variability: standards of measurement, physiological interpretation, and clinical use," Circulation, vol. 93, no. 5, pp. 1043-1065, 1996.

[37] R. Bailón, et al., "The integral pulse frequency modulation model with time-varying threshold: application to heart rate variability analysis during exercise stress testing," IEEE Trans Biomed Eng, vol. 58, no. 3, pp. 642-652, 2011.

[38] P. Laguna, G. B. Moody, and R. G. Mark, "Power spectral density of unevenly sampled data by least-square analysis: performance and application to heart rate signals," IEEE Trans Biomed Eng, vol. 45, no. 6, pp. 698-715, 1998.

[39] M. Orini, et al., "Characterization of dynamic interactions between cardiovascular signals by time-frequency coherence," IEEE Trans Biomed Eng, vol. 59, no. 3, pp. 663-673, 2012.

[40] P. Flandrin, "Time-frequency signal analysis and processing," chap. Ambiguity Function, pp. 160-167, Elsevier, 2003.

[41] M. Orini, et al., "A multivariate time-frequency method to characterize the influence of respiration over heart period and arterial pressure," EURASIP J Adv Signal Process, vol. 2012, no. 1, p. 214, 2012.

[42] K. F. Davis, K. P. Parker, and G. L. Montgomery, "Sleep in infants and young children: Part one: normal sleep," J Pediatr Health Care, vol. 18, no. 2, pp. 65-71, 2004.

[43] C.-L. Que, et al., "Homeokinesis and short-term variability of human airway caliber," J Appl Physiol, vol. 91, no. 3, pp. 1131-1141, 2001.

[44] A. L. Goldberger, "Fractal variability versus pathologic periodicity: complexity loss and stereotypy in disease," Perspect Biol Med, vol. 40, no. 4, pp. 543-561, 1997

[45] V.-P. Seppä, et al., "Tidal flow variability measured by impedance pneumography relates to childhood asthma risk," Eur Respir J, pp. ERJ00989, 2016.

[46] J. Veiga, et al., "Approximate entropy as a measure of the airflow pattern complexity in asthma," in Engineering in Medicine and Biology Society (EMBC), 2010 Annual International Conference of the IEEE, pp. 24632466, IEEE, 2010.

[47] V. Bellia, et al., "Relationship of nocturnal bronchoconstriction," Am Rev Respir Dis, vol. 140, pp. 363-367, 1989.

[48] R. Kurukulaaratchy, et al., "Predicting persistent disease among children who wheeze during early life," Eur Respir J, vol. 22, no. 5, pp. 767-771, 2003.

[49] F. Shaffer and J. Ginsberg, "An overview of heart rate variability metrics and norms," Front Public Health, vol. 5, p. 258, 2017.

[50] Y. Goren, et al., "Individual time-dependent spectral boundaries for improved accuracy in time-frequency analysis of heart rate variability," IEEE Trans Biomed Eng, vol. 53, no. 1, pp. 35-42, 2005.

[51] M. G. Tsipouras, D. I. Fotiadis, and D. Sideris, "An arrhythmia classification system based on the RR-interval signal," Artif Intell Med, vol. 33, no. 3, pp. 237-250, 2005

[52] T. W. Guilbert, et al., "Long-term inhaled corticosteroids in preschool children at high risk for asthma," $N$ Engl J Med, vol. 354, no. 19, pp. 1985-1997, 2006.

[53] G. Lezmi, et al., "Airway remodeling in preschool children with severe recurrent wheeze," Am J Respir Crit Care Med, vol. 192, no. 2, pp. 164171,2015

[54] A. D. Fryer and D. B. Jacoby, "Muscarinic receptors and control of airway smooth muscle," Am J Respir Crit Care Med, vol. 158, no. supplement_2, pp. S154-S160, 1998. 